\title{
La symbolique identitaire territoriale : reflet d'une culture manifeste Le cas québécois
}

\author{
Pierre Bérubé \\ Institut Québec-Europe : un patrimoine commun
}

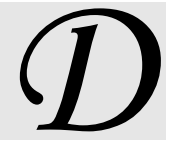

ans la chrétienté, l'expression du sentiment d'appartenance à l'État a toujours incité les sociétés à commémorer le "Saint » du pays en lui dédiant la basilique officielle de la patrie.

Ici, dans la capitale du Québec, le monument que constitue l'église Saint-Jean-Baptiste est déjà un objet de désistement ou de liquidation, qui sait?

Pratiquant ou non, deniers ou non, voilà donc des aboutissements et conclusions de notre culture particulière et contemporaine qui oublie ou renie.

\section{Identité, notion d'appartenance et spécificité}

Il est impossible d'examiner le fondement du concept de l'identité sans se référer obligatoirement à la notion foncière de l'appartenance. S'identifier, c'est avant tout «appartenir». Et appartenir, c'est recevoir, accepter et défendre toute une gamme de caractéristiques qui nous différencient et nous distinguent intrinsèquement.

En fait, on ne peut aborder le thème de l'identité sans se rapporter aux principes et aux rudiments qui conditionnent nos repères d'appartenance. Ce sont ces repères qui marquent, somme toute, notre particularité de la sorte. C'est donc à partir de cette façon d'être différent ou particulier que nous sommes constamment et instinctivement appelés à défendre, sauvegarder et garantir ces valeurs qui nous différencient de l'ensemble ou de "l'universalité ». Les besoins de développement (tant économique, social que culturel) et les relations de pouvoir qui s'ensuivent nous appellent à défendre nos intérêts, c'est-à-dire nos besoins et nos priorités basés sur notre condition différente et particulière.

\section{Différents modes d'expression identitaire}

Être particulier et appartenir (vouloir appartenir) à ses valeurs propres, cela concerne aussi bien l'individu qu'un groupe d'individus désirant se distinguer. L'individu, dès sa naissance, est appelé à développer les caractéristiques qui marquent son authenticité. Baptême, enregistrement et autres rituels de reconnaissance sont tous des gestes significatifs qui promulguent l'existence et l'appartenance exclusives de cette personnalité Ainsi, donner un nom, «appeler» quelqu'un, constitue la première impulsion qui initie l'existence propre de l'individu.

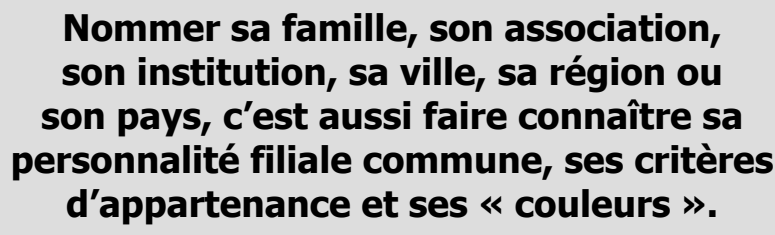

personnalité et leurs caractéristiques propres. Nommer personnalité et leurs caractéristiques propres. Nommer sa famille, son association, son institution, sa ville, sa région ou son pays, c'est aussi faire connaître sa personnalité filiale commune, ses critères d'appartenance et ses « couleurs ».

Depuis les origines de l'humanité, on peut se demander ce qui a inspiré l'élaboration de tant de manières d'exprimer le caractère distinctif de son espèce. En fait, l'environnement physique et psychique conditionne l'expression propre de l'entité et constitue l'indivisible source de référence. Cela est normal puisque l'entité qui désire exprimer son caractère particulier et 
sa marque de référence va chercher dans des valeurs qui l'entourent et la conditionnent celles qu'elle considère aptes et démonstratives pour la représenter ou la définir.

L'expression identitaire passe donc par une incommensurabilité de signes ou de gestes qui évoquent, pour une entité, les valeurs qu'on veut lui prêter. Ces signes figuratifs issus de l'environnement, du vécu ou de l'héritage de l'entité sont concrets ou abstraits, et même imaginaires. Ce qu'il faut donc discerner, c'est ce qu'ils veulent dire ou ce que l'on veut leur faire dire ${ }^{1}$. Ainsi, l'expression gestuelle (folklore, etc.), l'expression imagée (drapeaux, blasons, logos, etc.) ou l'expression auditive (chants, hymnes, slogans, cris de ralliement, etc.) constituent des représentations propres de référence et de convergence qui confirment et démarquent l'identité commune et consolident l'appartenance à une unité communautaire ou sociale quelconque.

\section{Et de là... la culture}

\section{Le bagage culturel : être ce qu'on est}

Du sentiment d'appartenance à ses valeurs, dont découlent les différentes expressions singulières de l'identité, s'exprime l'ensemble des caractéristiques qui définissent « la culture » d'une entité donnée. En fait, la culture est déterminée par tous les éléments spécifiques et collectifs qui caractérisent la personnalité d'une entité (nationale, régionale, locale, associative, individuelle, etc. $)^{2}$. Aborder l'analyse de la culture d'une entité, c'est vouloir décoder et expliquer le caractère distinctif qui l'individualise. Par conséquent, développer sa personnalité (personnalité culturelle), c'est soutenir, animer et accélérer l'évolution de ses valeurs propres.

\section{La culture versus l'éducation}

On ne peut prétendre qu'un individu ou une société «n'a pas de culture ». Nous sommes, en effet, tous dépositaires d'un bagage de valeurs et de notions qui modèlent et marquent notre personnalité. Celle-ci diffère des autres de par les caractéristiques propres qui l'ont érigée. Quelle soit pauvre ou riche, cela peut s'évaluer, s'apprécier ou s'estimer objectivement ou non. Mais on ne peut prétendre qu'une entité n'a pas de culture; il s'agit là d'un concept irrationnel. La richesse ou la pauvreté d'une quelconque entité et l'ex- pression distinctive de sa culture sont donc déterminées par la nature, la capacité et la qualité de l'environnement qui conditionnent son développement. Dans un environnement favorable, les valeurs de l'éducation et de la diffusion de la connaissance sont avantagées et consolident la santé et l'équilibre de l'entité. Dans un environnement pauvre, les moyens déclinent et ce sont les bases mêmes de la connaissance, de l'éducation et de la conscience de sa condition culturelle qui s'étiolent. C'est pourquoi, dans le langage populaire, lorsqu' on veut exprimer qu'une personne ou une communauté " n'a pas de culture », on désire tout simplement exprimer qu'il y a là un manque au niveau de la connaissance ou de l'éducation, ou une pauvreté dans l'expression littérale de sa personnalité.

\section{Tendance et force de dilution}

Outre les éléments intérieurs et spécifiques qui érigent la personnalité de l'entité et conditionnent son expression culturelle, il y a toute l'influence des autres composantes extérieures en relation avec celle-ci qui élargit le cadre typique de «l'exclusivité ». Les relations mutuelles et les échanges culturels permettent certes d'enrichir le milieu. Cela est incontestablement admis. Mais il est aussi démontré qu'une culture qui s'impose au détriment d'une autre la dégrade ou l'abâtardit. Lorsque l'échange n'a plus comme effet d'augmenter la teneur ou la valeur culturelle, mais au contraire a pour conséquence de détruire et d'altérer les fondements caractérisant la personnalité culturelle de l'entité, il s'agit d'un phénomène déstabilisant transposé sous différents vocables : assimilation, expansionnisme, colonialisme, etc. Les grandes tendances de masse telles la mondialisation ou l'américanisation (à titre d'exemples) sont des phénomènes effectifs actuellement en cause. Les mouvements de réorganisation de l'État ou de territoire initiés par différentes administrations (qui occasionnent le démantèlement ou la dilution de quelque façon des entités réelles) constituent aussi des forces immédiates de déstabilisation de celles-ci. Mutiler les frontières des entités culturelles ou les noyer avec d'autres entités hétérogènes sont des phénomènes souvent liés à des besoins dit de « rationalisation $»$ ou de $«$ relations de pouvoir $»^{3}$.

\section{Le cas québécois, une conjoncture notoire}

Si l'on avance qu'une entité réelle se définit par l'adjonction d'une foule de caractéristiques singulières, et 
que de son expression typique en découle une culture unique, on peut dire aisément que la culture québécoise procède d'une combinaison de circonstances tout à fait exclusives. Le rayonnement de sa culture en atteste la nuance et la coloration vis-à-vis de l'ensemble. Le but de cette analyse n'est pas d'en illustrer l'étalage. Mais le simple fait de souligner qu'il s'agit là d'une société de souche française, établie au nord d'un continent anglophone, régie par un code civil distinctif, à l'intérieur d'un système parlementaire britannique et limitrophe d'un voisin aussi influent que les USA illustre bien l'existence d'une société originale.

\section{Un rappel collectif}

On peut ainsi récapituler sommairement que l'environnement naturel québécois (de grands espaces, une hydrographie développée, un climat nordique, des ressources agricoles et forestières substantielles, un mode de production d'antan relié à diverses industries du domaine primaire), l'héritage culturel français et religieux ainsi qu'un système organisationnel de style britannique ont permis d'ériger les assises subjectives permettant de façonner un contexte référentiel propre à cette société globale qui, par son art et ses traditions, reflète une culture unique et solidaire.

Les forces positives de référence et l'appartenance ont fait croître des mouvements de solidarité tout à fait distinctifs et originaux. On n'a qu'à penser, entre autres, aux grandes corvées d'alors et à l'initiation au mouvement communautaire des Caisses populaires.

À l'opposé des forces mobilisatrices, la robustesse de la gouverne coloniale, qui imposa les façons de gérer et les valeurs de la mère patrie britannique, a érigé la réalité sociale par cette parodie qui esquisse la collectivité (canadienne-française) ainsi : "Le gouvernement et le commerce (business) sont du ressort des Anglais et la langue et la religion concernent les Français $» \ldots$.

C'est sur cette toile de fond condensé, c'est-à-dire :

- le développement des intérêts propres à sa société de souche originale;

- la sollicitude communautaire;

- l'adaptation ou la résistance relative à celle-ci quant aux codes et coutumes, modèles politiques et

économiques transmis par le pouvoir (colonial) supérieur;

- le nouvel ordre structurel de l'État à instituer;

que se sont tissées les assises du développement d'une culture nationale particulière.

C'est ainsi que les forces de consolidation propre par rapport aux forces de dilution dans un environnement respectif ont favorisé le caractère unique de l'entité québécoise et son expression originale au plan culturel.

\section{Les forces positives de référence et l'appartenance ont fait croître des mouvements de solidarité tout à fait distinctifs et originaux.}

\section{Une composante régionale jeune et indécise}

À partir de cette réflexion relative à l'identité et à la culture québécoise, on ne peut se référer seulement qu'à son aspect d'ensemble. Le Québec hérite de plusieurs composantes régionales dont l'environnement et le mode de production ont façonné nombre de collectivités respectives. Les caractéristiques géographiques ont érigé les us et coutumes de leurs habitants respectifs. De l'Abitibi à la Gaspésie et les Îles ainsi que de l'Estrie au Saguenay-Lac-Saint-Jean et le Nunavik, ce sont toutes des façons de faire et d'adaptation au territoire qui se sont développées dès lors.

Il faut soulever le cas des parties prenantes de l'entité québécoise. On a tendance à oublier que le développement de l'ensemble de la communauté nationale ne pourra cheminer qu'en mettant en valeur les caractéristiques propres des composantes du Québec global. Autant les forces de consolidation permettant l'union des intérêts communs et favorisant un développement intégré sont bonnes pour l'ensemble national, autant cela vaut pour une communauté régionale qui regorge de caractéristiques propres et rassembleuses.

Néanmoins, il faut préciser que l'établissement de l'écoumène sur le territoire québécois et le développement des différentes entités régionales datent d'une époque encore récente. Bien des régions en périphérie n'ont même pas encore atteint leur centenaire! De cette histoire nouvelle et d'une population relative- 
ment restreinte étalée sur un vaste territoire, peu de circonscriptions ou de divisions administratives ou politiques ont marqué par le passé l'organisation territoriale et la référence spatiale à cet égard. En fait, le comté municipal et le comté électoral (qui la plupart du temps se superposaient) ont été pendant longtemps (quelque $150 \mathrm{ans}$ ) le seul forum où les élus ont pu comparer leurs expériences. Les districts judiciaires et de recensement, par exemple, ont influencé bien marginalement l'organisation sociale et les populations. Il faut dire que le diocèse fut plus effectif à cet égard. On n'a qu'à penser à toutes les associations (souvent religieuses) et organismes créés à partir de l'entité diocésaine Les grandes régions administratives et la majorité des différents territoires d'intervention des ministères et organismes publics ne datent environ que d'une cinquantaine d'années.

Cela permet d'avancer que la notion de référence territoriale, les forces de cohésion régionales et l'évocation, voire la mémoire quant au sentiment d'appartenance au coin de pays demeurent un concept fragile. En fait, les constituantes intérieures ne sont pas encore consolidées, étant récentes, et les interminables réaménagements territoriaux et administratifs n'ont fait qu'activer l'hétérogénéité d'esprit à l'égard de la terre commune. Le sentiment d'appartenance à sa région et l'esprit d'identité qui conditionne la cohérence et l'adhérence, l'élan commun et le développement social intégré en sont directement affectés. Ce thème "régionaliste" sera traité de même manière dans cette analyse d'ensemble qui concerne l'identité et la culture québécoise.

L'expression de référence identitaire à sa culture propre constitue une force mobilisatrice tout à fait gratuite et renforce le vécu collectif, les affinités et les liens communs.

\section{La symbolique identitaire : outil de conviction et d'expression du sentiment d'appartenance}

Si le sentiment d'appartenance et sa manifestation s'expriment par l'expression des caractéristiques spécifiques d'une communauté - ce qui représente sa «culture »- la symbolique identitaire ${ }^{4}$ en esquisse ainsi la référence démonstrative de première ligne.

\section{Un dynamisme gratuit}

Depuis la nuit des temps ${ }^{5}$, afin de définir, marquer et préciser encore plus la singularité ou l'individualité de l'espèce, l'humanité définit et déploie une multitude de symboles qui dépeignent les traits, la nature et même les buts qui les conditionnent. Ils constituent des représentations de référence et de ralliement qui confirment et démarquent l'identité commune et qui réaffirment l'appartenance à la communauté ou à l'ensemble.

Ils répondent à ce besoin inné de montrer sa différence, de faire valoir sa spécificité et de défendre son authenticité et les valeurs qui assurent sa survie et ses fondements (sociaux, culturels, économiques, etc.). Ces outils de démonstration sont d'ailleurs davantage exploités dans les moments de compétition, de défense ou «lorsqu'il faut se faire valoir $»^{6}$. Par conséquent, l'expression de référence identitaire à sa culture propre constitue une force mobilisatrice tout à fait gratuite et renforce le vécu collectif, les affinités et les liens communs.

\section{Le phénomène nouveau de la symbolique à l'heure de la haute vitesse}

Jamais comme aujourd'hui la représentation symbolique n'a pris une telle dimension. Dans un monde de communication, de haute vitesse et de rationalisation des idées et des moyens, la consolidation des expressions et des messages s'ensuit. Un image (en l'occurrence un signe, un symbole) vaut mille mots, dit-on...

De l'Internet à bien d'autres moyens de diffusion expresse, les symboles d'expression identitaire (notamment les symboles de référence territoriale) se véhiculent à un rythme effarent. Ces moyens marquentils davantage le ralliement, l'appartenance et les références locales, régionales ou nationales ?

L'Internet, les nouvelles chaînes spécialisées de télévision ou la diffusion de l'information, de la culture ou de l'éducation par vidéocassettes font partie des nouvelles pratiques culturelles de développement. Cette nouvelle complémentarité des médias contribue à façonner ce nouveau paysage socioculturel. Au point d'ailleurs que certaines frontières auxquelles nous nous étions habitués tendent à s'estomper.

Dans ce contexte, assiste-t-on à un phénomène d'affaiblissement ou de brouillage de nos frontières de 
référence, ou s'agit-il plutôt d'un renouvellement des outils de diffusion? Comment se traduit la représentation symbolique par rapport aux territoires d'appartenance locaux, régionaux ou nationaux ? Chose certaine, l'utilisation du symbole dans notre monde moderne devient davantage un outil d'expression tangible, rapide et efficient ${ }^{7}$.

\section{Le Québec : expression culturelle distincte et expression symbolique trouble}

Qui exprime son nom et dévoile sa bannière invoque automatiquement une référence relative au sentiment d'appartenance à sa culture. Qu'en est-il donc de l'image ou de la représentation, au Québec particulièrement durant cette période de transformation, des méthodes de diffusion et des nouvelles relations et communications virtuelles et à haute vitesse ? Si l'on se fie à ce qu'on perçoit actuellement tant au plan national que régional, on peut facilement déduire que l'ambivalence s'exprime à souhait.

Depuis plus de deux siècles, notre société se voit diriger simultanément par différents niveaux de gouvernement ou d'administration (fédéral, provincial, régional divers, supra municipal et municipal), et sans cesse elle doit assimiler ces références identitaires. Tant au plan de l'octroi des services qu'à celui de la représentation ou de la participation aux responsabilités civiles, notre sentiment d'appartenance nationale, régionale et locale est sollicité. Les résultats d'études et de sondages divers nous confirment cet embrouillamini dans l'expression de notre sentiment d'appartenance.

\section{L'influence de la symbolique comme facteur de référence identitaire devient davantage un outil de sensibilisation et de mobilisation à l'intérieur de nos sociétés modernes et relationnelles.}

Cette emphase qu'ont nos voisins du Sud à exposer, d'une façon naturelle et spontanée, leurs symboles d'identité nationale ou régionale parallèlement à notre relative contingence ou gêne à exercer un même type de manifestation, justifie en soi un questionnement. L'enchevêtrement facilement perceptible des messages de référence nationale ou régionale est aussi un indice qui caractérise une forme de particularité de notre expression identitaire. L'analyse cherche à démystifier la situation et veut apporter une réflexion sur ces circonstances qui conditionnent ce comportement singulier concernant notre référence identitaire globale et régionale.

\section{Un rappel historique d'une symbolique embrouillée}

L'expression culturelle du fait distinctif de la société québécoise s'exprime par delà les années par une symbolique aussi embrouillée que déroutante. Il faut croire qu'une population pareillement partagée quant à son appartenance nationale se reflète aussi par une symbolique pareillement multiforme ${ }^{8}$. Ce bref rappel des étapes relativement récentes de l'expression symbolique et officielle du Québec nous montre la déficience concernant l'expression de la réalité québécoise via les manifestations publiques et conventionnelles désignant le peuple et la culture québécoise.

Ainsi, il faut se souvenir qu'il y a à peine soixante ans, la bannière britannique flottait encore sur le parlement du Québec et les ministères et organismes du « gouvernement provincial». L'Union Jack constituait le symbole identitaire de l'État. Pendant presque 200 ans, l'ambiguïté s'inaugurait et s'organisait sur ce plan au Québec. Pour les Anglophones du Canada, cette symbolique constituait une prolongation normale de l'appartenance à la mère patrie' ${ }^{9}$. Cependant, pour les francophones du Québec, l'anachronisme identitaire s'instituait et l'on devait commencer illico à «apprendre » à biaiser un aspect fondamental et officiel de notre expression collective et culturelle.

Comme preuve flagrante que cette dissymétrie ne pouvait tenir ou continuer, les symboles nationaux canadiens - absolument fondamentaux (tels le drapeau unifolié et l'hymne national «Ô Canada ») - furent empruntés du terroir ou de l'héritage de cette même communauté francophone en manque de «rationalité » symbolique à cet égard.

En outre, malgré l'adoption d'un drapeau québécois en 1948, les Québécois ne se sont jamais véritablement remis de ces dispositions politiques du passé qui les ont amenés à accepter des symboles et des attributs devant les représenter, mais qui ne reflétaient en rien leur personnalité ou leur identité culturelle.

Il faut se rappeler que, dans les années 1960 et 1970, il n'y a pas si longtemps, lorsqu'on voulait souligner 
les événements officiels (fêtes, parades, assemblées, etc.), on pavoisait indiciblement avec divers drapeaux et étendards combinés : le drapeau bleu avec le « $\mathrm{M}$ » de Marie s'entrecroisait avec le fleurdelisé, le drapeau du Sacré-Cœur avec l'Union Jack, le Red Ensign avec le drapeau du Vatican, etc. Le besoin inné d'expression et d'appartenance culturelles retentissait bien, mais la compréhension de la signification symbolique en liesse ne constituait qu'un vide référentiel en soi.

Par surcroit, qu'en est-il de toutes ces références symboliques monarchiques qui rappellent doucereusement un passé dépréciatif et minoratif de notre société et qui évoquent les souvenirs d'une circonstance de défaite et de soumission historique?

Se donner des symboles représentatifs permet officiellement de se confirmer et «d'exister ». Or, aucune loi québécoise ne peut être en vigueur si elle n'a pas reçu la sanction royale. Des signes aussi palpables que la monnaie, les lettres de créances, les timbres à l'effigie de la Souveraine, les terres de la Couronne, la Gendarmerie royale, la Cour du Banc de la Reine, etc. ne sont que quelques manifestations qui expriment bien clairement cette réalité contrefaite.

Par conséquent, au début des années 2000, qu'en estil de cette représentation majeure qui transcende encore la réalité québécoise, contrarie sa personnalité, exige des fonds publics et fige comme depuis toujours l'aberration comme norme et cela, à une époque où l'âge colonial est depuis longtemps révolu?

\section{La résonance contemporaine}

De cette forme de «soumission symbolique », devenue pratiquement la norme, ne faut-il pas se surprendre aujourd'hui que toute discussion et même débat sur le pavoisement ou autre type d'expression nationale réveille chez nous le complexe identitaire, ce vieux système défectueux devenu tabou?

L'influence de la symbolique comme facteur de référence identitaire devient davantage un outil de sensibilisation et de mobilisation à l'intérieur de nos sociétés modernes et relationnelles. Les événements du 11 septembre aux États-Unis et les dernières élections présidentielles en France ont été sans conteste les plus récentes manifestations d'envergure qui ont témoigné de l'importance de ce phénomène de rassemblement par l'émission de symboles de références au territoi-

$\mathrm{re}^{10}$. Ici, on se confond dans l'éteignoir, le neutralisme et l'option rassurante du «pas de chicane de drapeaux!».

\section{On peut se demander comment un développement culturel permettant la mise en valeur des intérêts et des particularités communes pourra se faire si l'on n'arrête pas d'embrouiller les références à la communauté régionale.}

\section{Une tendance persistante et démobilisatrice}

Dénommer et se donner des signes et des symboles caractéristiques, c'est vouloir consolider et garantir sa spécificité. C'est le sommet, la confirmation ultime et l'attestation manifeste et «palpable » de sa personnification (individuelle, familiale, régionale, nationale, commerciale, institutionnelle, sociale, etc.).

La confirmation ou la ratification représentative de l'entité québécoise et de ses composantes régionales, telle qu'énoncée, a donc subi des insuffisances, sinon des absences dans le passé. Il s'agit d'un germe de dilution ou de brouillage des esprits de référence qui altère la solidarité. Ce problème d'identité devient pratiquement une caractéristique spécifique de ce Québec distinct qui persévère dans ce dispositif d'inconscience et de désordre émotif au plan culturel.

- Au plan d'ensemble de la société québécoise, la référence à la nation devient davantage ambiguë. De la Commission de la capitale nationale à Québec à la Commission de la capitale nationale à Ottawa, des parcs nationaux du Québec aux parcs nationaux fédéraux, de la fête nationale du Québec à la fête nationale du Canada, de l'Ordre national du Québec à l'Ordre national du Canada, etc., on ne sait plus très bien où en est la nation. Si l'on ajoute en boutade le paradoxe de l'adoption des lois « d'ici » sanctionnées par la représentante de la Couronne britannique, il y a de quoi se questionner sur les reflets et symboles fondamentaux de l'identité culturelle québécoise.

- Au plan régional, les enchevêtrements administratifs, les redécoupages territoriaux et les redésignations des entités organisationnelles n'en finissent plus. On peut se demander comment un développement culturel permettant la mise en valeur des inté- 
rêts et des particularités communes pourra se faire si l'on n'arrête pas d'embrouiller les références à la communauté régionale.

En une vingtaine d'années environ, on a érigé les MRC qui avaient certes des racines historiques solides; on a aussi désigné de nouvelles régions administratives, dessiné et appelé de nouveaux territoires de commissions scolaires qui n'ont rien à voir avec les précédentes. La Commission de la représentation électorale du Québec redéfinit le territoire des circonscriptions après chaque élection (prévu dans la loi) et reconditionne l'appartenance des populations indéfiniment ${ }^{11}$. Et il y a aussi la même commission au plan fédéral qui poursuit le même processus. Et que dire des derniers mouvements de regroupements municipaux qui ont vu poindre une pléthore de nouvelles désignations relativement aux arrondissements, districts et même des municipalités aussi importantes que Chicoutimi ou Hull, à titre d'exemple?

De l'appartenance à un même coin de pays au centre du Québec, appelé Amiante, Bois-Francs, Estrie, Cantons de l'Est ou Centre-du-Québec ${ }^{12}$, il y a de quoi donner un vertige référentiel. Il n'y a pas si longtemps, la Mauricie fut appelée « Centre-du-Québec » au plan administratif et « Cœur-du Québec » pour les fins de promotion touristique du gouvernement $\mathrm{du}$ Québec. Il ne s'agit encore là que d'un exemple démontrant l'enchevêtrement identitaire, même au plan de l'appellation. C'est ainsi que la superposition des messages identitaires ou des symboles de référence hétérogènes se développe et entremêle les esprits de différentes façons.

\section{Communauté, mémoire, fidélité}

Enfin, une communauté nationale ou régionale qui néglige de telle façon une facette aussi importante de sa personnalité culturelle vient à délaver ses références et ses racines mobilisatrices et représentatives. Le sentiment d'appartenance, " ce ciment communautaire ", doit faire l'objet d'une sensibilisation accrue auprès des autorités qui ont une influence ou une responsabilité vis-à-vis de la détermination, de la désignation et de la reconnaissance d'une entité représentative et distinctive. Cette lecture sur la symbolique identitaire territoriale est au cœur même des choix et des outils concrets favorisant la solidarité, l'union des intérêts communs et le développement de la culture propre à l'entité.
Il est important de savoir, de comprendre et de statuer sur ce qu'est sa communauté; signifier, valoriser et enseigner sa mémoire et ses racines; et inculquer aux dirigeants qui décident et statuent sur ces balises de rechercher ou de poursuivre un esprit de fidélité concernant ces facteurs de référence qui cautionnent l'entité culturelle (et ne pas la diminuer).

La symbolique identitaire est devenue au Québec un outil incontournable et nécessaire pour contrer cette disposition sociale encline à la nonchalance et à l'abdication quant à l'esprit de référence à son nom, sa collectivité et son intégralité culturelle. Il est temps de se sensibiliser et de réagir au nom de soi-même.

\section{Notes biographiques}

Originaire de Cabano au Témiscouata, Pierre Bérubé possède actuellement ses assises à Québec à l'Institut Québec-Europe à titre de secrétaire-général. Il est détenteur d'un doctorat en urbanisme (Académie de droit, d'économie et des sciences d'Aix-Marseille III), d'une maîtrise en aménagement régional (Université Laval), d'un baccalauréat spécialisé en sciences politiques (Université d'Ottawa) et d'un baccalauréat ès Arts en histoire (Université d'Ottawa).

\section{Notes et références}

1 Ex. : Le croissant de lune islamique, la feuille d'érable canadienne, les anneaux olympiques, la devise « Je me souviens », l'hymne «La Marseillaise », la ceinture fléchée, le globe stylisé de l'ONU, etc.

2 L'ensemble de toutes les valeurs déterminées par le cadre environnemental de vie d'une entité et véhiculées au cours de l'histoire par la mémoire collective constitue le « fourniment » caractéristique de cette entité. Son expression par le langage, l'art et la façon de faire respective constitue sa culture propre.

3 Cf. : Bérubé, P. (1983), Le Témiscouata en toute justice, Presses de l'Université du Québec (GRIDEQ), p. 111-113; (1993), L'organisation territoriale du Québec - Dislocation ou restructuration? - Urgence d'agir, Les Publications du Québec, p. 99.

4 Tout symbole signifiant et exprimant l'appartenance ou la référence (exemples déjà proposés).

5 Déjà, dans 1'Ancien Testament ou la Torah, dès le XIII siècle avant J.-C., « Yahvé parla à Moïse et à Aaron, en disant : les fils d'Israël camperont chacun sous sa bannière, sous les enseignes de leurs maisons patriarcales. [...] Le 
départ [pour la Terre promise] [...] aura lieu chacun à sa place selon sa bannière $[\ldots]$, c'est ainsi qu'ils campèrent selon leurs bannières $[\ldots] »$, etc.

6 On n'a qu'à penser aux compétitions sportives (notamment les Jeux olympiques) qui deviennent de plus en plus des spectacles de promotion étatique.

Le gouvernement fédéral l'a peut-être compris plus que d'autres dans sa campagne de promotion de l'unité nationale qui a donné lieu à certains dérapages autour de l'affaire des « commandites ».

\footnotetext{
Fragmentée, distribuée ou prodiguée de diverses façons.
}

9 Soulignons que l'Union Jack est encore un des deux drapeaux officiels du Canada avec l'Unifolié.

${ }^{10}$ La bannière étoilée et le Tricolore.

11 À titre d'exemple, le précédent redécoupage effectif en 2003 a fait disparaître le nom de « Limoilou » pour « JeanLesage » et « Saguenay » pour René-Lévesque ». Les références territoriales réelles (Limoilou, une constituante authentique de la ville de Québec et la rivière Saguenay qui longe physiquement ledit comté) ont fait place à ces improvisations dénominatives qui n'ont rien à voir avec les communautés qui vivent leur territoire.

12 Désignation de la nouvelle région administrative sur la rive sud du Saint-Laurent (Drummondville). 